\title{
Attendance, engagement and performance in a medical school curriculum: Early findings from competency-based progress testing in a new medical school curriculum
}

\author{
Heather Laird-Fick $^{1}$, David J Solomon ${ }^{\text {Corresp.. }}{ }^{2}$, Carol Parker ${ }^{3}$, Ling Wang ${ }^{1}$ \\ 1 Department of Internal Medicine, Michigan State University, East Lansing, Michigan, United States \\ 2 Office of Medical Education Research and Development, Michigan State University, East Lansing, MI, United States \\ 3 Academic Affairs, Michigan State University, East Lansing, Michigan, United States \\ Corresponding Author: David J Solomon \\ Email address: dsolomon@msu.edu
}

Introduction Medical students often do not value attending in-person large group sessions. It is also not clear from prior research whether attendance at large group sessions impact on performance in medical school. The goal of this study was to assess the relationship between voluntary attendance in large group sessions organized as a "flipped classroom" in a new innovative curriculum and students' mastery of clinical applications of basic science knowledge.

Methodology Our students' ability to apply basic science knowledge to clinical problems is assessed via progress testing using three methodologies: a locally developed multiple-choice examination, written examination developed through the National Board of Medical Examiners (NBME) Customized Assessment Services Program and post encounter questions included in a clinical skills examination. We analyzed the relationship between voluntary attendance at weekly large group "flipped classroom" sessions and the students' performance on examinations given at four intervals over the initial 24-week module of the medical school curriculum.

Results Complete data were available for 167 students. A total of 82 students $(49.1 \%)$ attended all large group sessions, 65 students (38.9\%) missed one or two sessions and 20 students (12.0\%) missed 3 or more sessions. There were no difference between the students in the groups on their medical admission (MCAT) examination scores. The growth in performance from each time point until the next was statistically significant. There was no statistically significant difference in growth between the students who had no absences and those who had one or two absences. Students who missed 3 or more sessions performed significantly lower than their peers over the 24 week module and were more likely to score one or more standard deviations below the class mean on the assessments.

Conclusions We found no relationship between attendance and MCAT scores suggesting the differences in performance on the progress tests was not due to initial differences in knowledge or reasoning skills. While the study was not experimental, it suggests large group sessions using a "flipped classroom" approach to provide reinforcement, feedback and practice may be effective for increasing learning and retention in the application of basic science knowledge among first year medical students. 
1 Title: Attendance, engagement and performance in a medical school curriculum. Early findings from competency-based progress testing in a new medical school curriculum

Authors: $\quad$ Heather Laird-Fick 0000-0001-9215-8152

Dept. of Medicine, Michigan State University College of Human Medicine, East Lansing, Michigan, United States

Heather.lairdfick@hc.msu.edu

David J Solomon 0000-0002-3130-5240

Office of Medical Education Research and Development, Michigan State,University College of Human Medicine, East Lansing, Michigan, United States

dsolomond@msu.edu

Carol Parker 0000-0003-4934-4325

Academic Affairs, Michigan State University College of Human Medicine, East Lansing, Michigan, United States parkerca@msu.edu

Ling Wang 0000-0003-2452-6691 Dept. of Medicine, Michigan State University College of Human Medicine, East Lansing, Michigan, United States Ling.wang@hc.msu.edu

Running Title: Attendance, engagement and performance in medical school 
Abstract

Introduction Medical students often do not value attending in-person large group sessions. It is also not clear from prior research whether attendance at large group sessions impact on performance in medical school. The goal of this study was to assess the relationship between voluntary attendance in large group sessions organized as a "flipped classroom" in a new innovative curriculum and students' mastery of clinical applications of basic science knowledge.

Methodology Our students' ability to apply basic science knowledge to clinical problems is assessed via progress testing using three methodologies: a locally developed multiple-choice examination, written examination developed through the National Board of Medical Examiners (NBME) Customized Assessment Services Program and post encounter questions included in a clinical skills examination. We analyzed the relationship between voluntary attendance at weekly large group "flipped classroom" sessions and the students' performance on examinations given at four intervals over the initial 24-week module of the medical school curriculum.

Results Complete data were available for 167 students. A total of 82 students (49.1\%) attended all large group sessions, 65 students (38.9\%) missed one or two sessions and 20 students $(12.0 \%)$ missed 3 or more sessions. There was no difference between the students in the groups on their medical admission (MCAT) examination scores. The growth in performance from each 
46 time point until the next was statistically significant. There was no statistically significant

47 difference in growth between the students who had no absences and those who had one or

48 two absences. Students who missed 3 or more sessions performed significantly lower than their

49 peers over the 24 week module and were more likely to score one or more standard deviations

50 below the class mean on the assessments.

51

52 Conclusions We found no relationship between attendance and MCAT scores suggesting the

53 differences in performance on the progress tests was not due to initial differences in knowledge

54 or reasoning skills. While the study was not experimental, it suggests large group sessions using

55 a "flipped classroom" approach to provide reinforcement, feedback and practice may be

56 effective for increasing learning and retention in the application of basic science knowledge

57 among first year medical students. 
59

60

61

62

63

64

\section{Background}

In the fall of 2016 Michigan State University's (MSU) College of Human Medicine (CHM) implemented a new curriculum called the Shared Discovery Curriculum (SDC). The SDC is organized around patient chief complaints and concerns and student assessment is done via progress testing. Progress testing uses parallel forms of a very broad-based examination given multiple times over an extended course of study and is well suited for problem-based learning curricula such as the SDC. (Van Der Vlueten, Verwijnen \& Wijnen, 1996) A detailed description of the SDC curriculum and the Progress Suite of Assessments are available on the SDC website (MSU, 2015)

The Early Clinical Experience (ECE) constitutes the initial 24-week module of the SDC. Students are trained in basic data gathering and patient communication skills and after eight weeks begin working in clinic settings with medical assistants and nurses. They also begin mastering clinical applications of basic science knowledge through guided independent study, problem-solving exercises in small groups, and in weekly large group sessions. The large group sessions use a flipped classroom model, during which the students apply basic science concepts they studied over the preceding three days. Attendance at the large group session was encouraged but not required during the first year the curriculum was implemented. the increasing availability of educational resources designed to help them prepare for the United States Medical Licensure Examination (USMLE) Step 1. (Zazulia \& Goldhoff, 2014) Research on the relationship between classroom attendance and academic performance in 
80

81

82

medical school is mixed. Fogleman \& Cleghorn (1983) reported an association between selfreported class attendance and National Board of Medical Examiner (NBME) Part I performance. Other studies in health education, however, have not found a relationship between attendance and performance. (Azab et al, 2015; Eisen et al, 2015) The relationship between attendance and grades among undergraduate college students however was found to be quite strong and relatively independent of other predictors in a large scale meta-analysis. (Credé, Roch \& Kieszczynka, 2010) The lack of consistent findings in health professions education could be do to a number of factors. There are relatively few studies, most small scale and with differing conditions.

As part of the evaluation of the SDC we assessed the relationship between attendance in the large group sessions of the ECE and performance on the progress tests given over the first year of the curriculum. The goal of this study is to assess the relationship between voluntary attendance in the ECE large group sessions and the students' mastery of clinical applications of basic science knowledge in the SDC and help determine whether it would be prudent to require attendance in these sessions in the future.

\section{Methods}

materials were provided to the students three days prior to an LGA session. To assess how well the students had prepared for the LGA, they took an Individual Readiness Assessment Test (IRAT) at the beginning of each LGA session. The IRAT was used to provide formative feedback and not for grading purposes. We used a score of zero on the IRAT as a proxy measure to 
101 identify students that did not attend the large group session that week. Each IRAT had

102 between 28 and 50 points and it is highly unlikely a student who completed the test would have

103 a legitimate score of zero. Students were categorized into three groups: (1) those who

104 attended all sessions, (2) those who missed one or two sessions and (3) those who missed three

105 or more sessions. With 24 sessions over the course of the ECE, we hypothesize it would be

106 quite possible for a student who intended to attend all the large group sessions to miss one or

107 two sessions for reasons largely beyond their control, such as an illness or family emergency.

108 We also hypothesized that when students missed more than two sessions they probably do so

109 as a conscious decision that their time would be better spent in other activities rather than for

110 reasons beyond their control. Given that each LGA focused on the application, rather than the

111 delivery, of new content, the students' primary penalty for missing an LGA would be to miss an

112 opportunity to receive additional practice and formative feedback on the concepts covered that

113 week.

As part of the Progress Suite of Assessments (PSA), students' ability to apply basic science knowledge to clinical problems is assessed using three methodologies. Students complete the PSA at four points during the 24-week ECE; shortly after matriculation in late

117 September, mid-November, mid-February of the following year and at the end of the ECE in 118 April of the following year. developed and consists of 140 items from a large item pool used in the previous curriculum as well as additional items created to ensure comprehensiveness. While each of the four 
122 administrations included different items, they were designed to be roughly equivalent though

123 they were not equated through a formal psychometric process. The second PSA was taken at

124 about the same interval and was designed through the National Board of Medical Examiners

125 (NBME) Customized Assessment Services Program. ${ }^{a}$ While slightly different domains of content

126 were included in each of the four administrations, we have treated the different versions as

127 roughly equivalent examinations. Along with written examinations, students take a Progress

128 Clinical Skills Examination (PCSE) that includes a post encounter component designed to assess

129 students' ability to apply basic science knowledge to the standardized patient interaction they

130 just completed. There were eight cases in each PCSE examination. The number and nature of

131 the items varied from case to case. For example, the student may have to write a note about

132 the patient or answer some questions about the basic science underlying the patient's

133 complaint. Gold and his colleagues describe the PCSE in more detail. (Gold et al, 2015) The

134 percentage of items correct was used as the outcome measure in both sets of written

135 examinations. The percentage of possible points that could be achieved in the post encounter

136 stations was used as the outcome measure for the post encounter stations.

We assessed the relationship between large group attendance and the three

performance measures in two ways. First, we conducted a repeated measure analysis of

variance (ANOVA) for the three PSAs with the four administrations of these assessments as the

design over measures and the categorized number of missed large group sessions as the design

over subjects. We used planned comparisons to test for differences in the levels of each factor.

142 For the design over measures we tested the significance of the change in score from the first

a http://www.nbme.org/schools/cas.html 
143 administration to the second, the second to the third and the third to the fourth. For the

144 absence categories, we tested for a difference between students with no absences and

145 students with one or two absences and then for a difference between students with two or

146 fewer absences and students with three or more absences.

147 Secondly, we compared the number of times a student scored at least one standard

148 deviation (SD) below the mean for each of the three measures across all four time points (total

149 of 12 assessments) for the three groups of students. We used a score of one SD or more below

150 the mean as an indicator that a student's performance was well below their peers on that

151 assessment and may need remediation. We categorized this measure into students who did not

152 have any scores at least one SD below the mean, those who had one to four scores one or more

153 SD below the mean and those who had more than four scores one or more SD below the mean.

154 The relationship between the categorized number of absences and categorized number of

155 assessments where the student scored one or more SD below the mean was tested by Fisher's

156 exact test. As with the repeated measures we conducted two comparisons, students without

157 any absences with those who had one or two absences; and students who had less than three

158 absences with those who had three or more absences.

We also compared the students' Medical College Admissions Test (MCAT) total scores among the students in the three absence groups. The exam is a written multiple-choice test taken by medical school applicants that assesses critical analysis and reasoning skills along with relevant basic science and behavioral knowledge. We used a one-way analysis of variance to

163 test for statistically significant differences among the students in the three attendance groups. 
We used SPSS Version 24 to conduct the statistical analyses and considered statistical

165

166

167

168

169

170

171

172

173

174

175

176

177

178

tests where $p<0.01$ as statistically significant. An "honest broker" was used to provide deidentified data to conduct this study. The use of an honest broker to provide deidentified and hence not human subject data has been approved by the Michigan State University Research Protection Program.

\section{Results}

Complete data were available for 167 students. A total of 82 students (49.1\%) attended all large group sessions, 65 students (38.9\%) missed one or two sessions and 20 students $(12.0 \%)$ missed three or more sessions. We compared the MCAT scores of the three groups above to assess if they entered medical school with different levels of basic/behavioral knowledge or reasoning skills. We found essentially no differences in MCAT performance among the students in the three groups. The total MCAT scores were $506.48 \pm 5.00$, $506.44 \pm 5.54$, and $506.56 \pm 5.16$ (mean \pm SD) for the group with no missed sessions, one or two missed sessions and the three or more missed sessions. The differences were not statistically significant.

Tables 1 presents summary statistics over the four administrations and within the three absence categories for the NBME customized examination, locally developed examination, and the post encounter component of the PCSE. Figures 1, 2 and 3 present these data graphically. Based on the repeated measures ANOVAs, there was no statistically significant interaction between the categorized number of absences and the change over time in student performance for any of the PSAs although the interaction for the NBME and the post 
185

186

187

188

189

190

191

192

193

encounter component of the PCSE approached statistical significance at $(p=0.02)$ and $(p=$

0.03) respectively. For all three PSAs, the growth in performance from each time point until the next was statistically significant $(p<0.01)$ except for the post encounter component of the PCSE where the change Feb. 2017 to Apr. 2017 approached statistical significance $(p=0.03)$. Also, for all three PSAs, there was no statistically significant difference between the students who had no absences and those who had one or two absences. There was a statistically significant difference $(p<0.01)$ between students who had two or fewer absences and those who had three or more absences with the students with fewer or no absences performing significantly better than those with 3 or more absences.

Table 2 presents a crosstabulation of the categorized number of times a student scored one SD or more below the mean by the categorized number of absences. We found no statistically significant difference in the categorized number of scores one or more SD below the mean for students with no absences and those with one or two absences. Students with three or more absences had more scores that were one or more SD below the mean than students with two or less absences $(p<0.01)$.

\section{Discussion}

ECE experience. Given this is the first year of a newly implemented curriculum these findings are encouraging. Students who chose to attend 22 or more of the 24 weekly large group sessions performed better on all three PSAs and were considerably less likely to receive scores one or more SD below the mean when compared with students who had three or more large 
206 group session absences. These differences persisted across the 24 -week module. While

207 students with higher attendance rates out performed students with lower attendance, there

208 was no differences in MCAT scores among the students in the three attendance groups. This

209 suggests the differences in performance on PSAs do not reflect initial differences in basic and

210 behavioral science knowledge or reasoning skills which the MCAT is designed to measure.

211 There also was no interaction between growth over time and absences suggesting that while

212 students with three or more absences did not performed as well as students with less than

213 three absences initially, the gap did not widen or narrow at least to the point where it was

214 statistically significant.

The large group sessions used a "flipped classroom" approach where new material was

not introduced. They instead provided reinforcement, feedback and practice for the material already introduced to the students in their individual and small group curriculum. There is evidence that this approach to curriculum design my be more effective than the traditional teacher-centered approach to large group instruction for learning essential basic science material (Street et al, 2015) as well as increasing student satisfaction and engagement in the curriculum. (McLaughlin, 2014) This approach is also consistent with a social constructivist perspective on learning which emphasizes the importance of discussion and interaction with other students and teachers in the learning process. (Palinscar, 1998) performance in medical school and the findings are mixed in the few studies done. More research has been done on students at the undergraduate college level. Credé, Roch and 
227 Kieszczynka (2010) conducted a meta-analysis of undergraduate college students that provides

228 a comprehensive summary of a large body of research on the relationship between class

229 attendance, grades and other student characteristics. It is not clear whether their finding can

230 be applied to medical students, but it seems reasonable to consider their finding in interpreting

231 the results of our study. As in our study, Credé and his colleagues (2010) found a moderate

232 relationship between attendance and performance. In their meta-analysis attendance

233 correlated $p=0.44$ with course GPA and $p=0.41$ with overall college GPA which was more

234 predictive than SAT scores, high school grades or surveys of study habits/skills.

235 Since our study was not experimental we cannot be sure the relationship between

236 attendance in the large group sessions and performance on the PSAs is causal. The relationship

237 between attendance and the PSAs may be mediated by some third factor or factors such as

238 conscientiousness, motivation and/or study skills/habits. Credé and his colleagues' (2010)

239 meta-analysis suggested this was not the case for the relationship between class attendance

240 and grades in undergraduate students. While it is not clear the relationship between

241 attendance in the large sessions and performance on the PSAs is causal, the evidence was a

242 major consideration in our medical school's decision to start requiring students to attend the

243 large group sessions in the ECE. It should also be noted that this study was based on a single

244 year's worth of data and this was the first year of a new curriculum.

245 Conclusions

246 Our study found that students who voluntarily attended all or most large group "flipped classroom"

247 teaching sessions in the first 24-week block of our medical school curriculum performed significantly 
248 better on progress skills assessment examinations. We found no relationship between attendance and

249 MCAT scores suggesting the differences in performance on the PSAs was not due to initial differences in

250 knowledge or reasoning skills which the MCAT is designed to assess. While the study was not

251 experimental, it suggests large group sessions using a "flipped classroom" approach to provide

252 reinforcement, feedback and practice may be effective for increasing learning and retention among first

253 year medical students.

254

255 
259

260

Azab E, Saksena Y, Alghanem T, Midle JB, Molgaard K, Albright S, Karimbux N. Relationship

261 Among Dental Students' Class Lecture Attendance, Use of Online Resources, and

262 Performance. Journal of Dental Education 2015;80:4, 452-8.

263

264

Credé M, Roch SG, Kieszczynka UM. Class Attendance in College: A Meta-Analytic Review of the Relationship of Class Attendance with Grades and Student Characteristics. Review of

266 Educational Research 2010 June 80(2):272-95 doi: 10.3102/0034654310362998

267

268

Eisen DB, Schupp CW, Isseroff RR, Ibrahimi OA, Ledo L, Armstrong AW. Does class

269 attendance matter? Results from a second-year medical school dermatology cohort study Int J Dermatol. 2015 Jul;54(7):807-16. doi: 10.1111/ijd.12816.

271

272 Fogleman BS, Cleghorn GD Relationship between class attendance and NBME Part I Examination. Journal of Medical Education 1983;58:10, 904.

274

275

276

Gold J, DeMuth R, Mavis B, Wagner D. Progress testing 2.0: clinical skills meet necessary

277 science. Med Educ Online 2015; 20:1 retrieved 2017-06-01 from http://dx.doi.org/10.3402/meo.v20.27769

278

McLaughlin JE, Roth MT, Glatt DM, Gharkholonarehe N, Davidson CA, Griffin LM Esserman DA, Mumper RJ. The Flipped Classroom: A Course Redesign to Foster Learning and Engagement in a Health Professions School Acad Med. 2014;89:236-243 doi: 10.1097/ACM.0000000000000086 
287 Palinscar AS. Social constructivist perspectives on teaching and learning. Annu Rev Psychol 288 1998;49:345-75.

289

290 Street SE, Gilliland KO, McNeil C. Royal K. The Flipped Classroom Improved Medical Student 291 Performance and Satisfaction in a Pre-clinical Physiology Course Med.Sci.Educ. (2015)

292 25:35-43. DOI 10.1007/s40670-014-0092-4

293

294 Van Der Vlueten CPM, Verwijnen GM, Wijnen WHFW. Fifteen years of experience with 295 progress testing in a problem-based learning curriculum. Medical Teacher 1996;18:2;103-9. 296

297 Zazulia AR \& Goldhoff P Faculty and Medical Student Attitudes About Preclinical Classroom 298 Attendance, Teaching and Learning in Medicine 2014;26:4, 327-34, DOI:

$299 \quad 10.1080 / 10401334.2014 .945028$. 
Figure 1 (on next page)

NBME performance by number of large group absences 


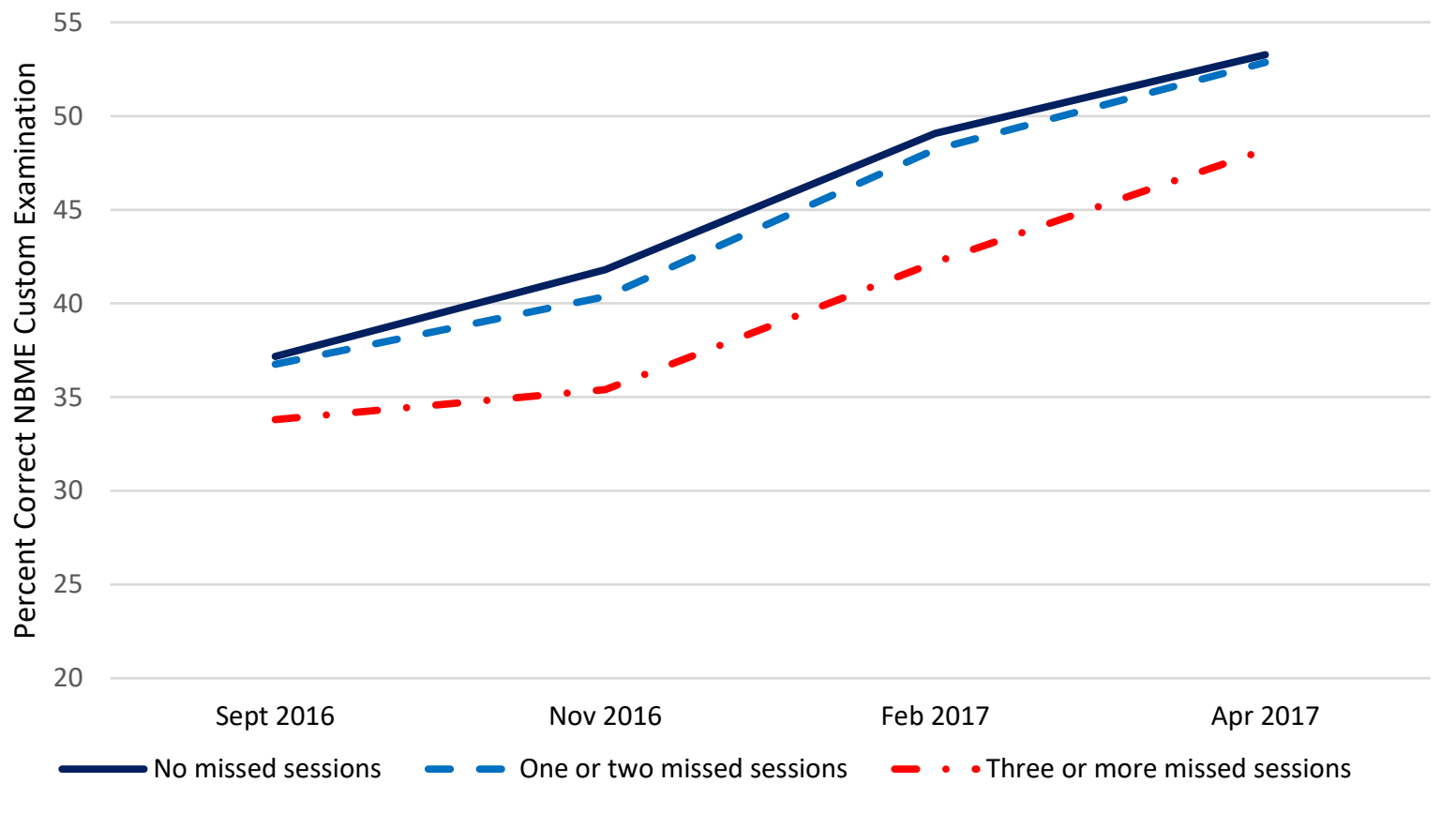




\section{Figure 2 (on next page)}

Locally developed performance assessment by number of large group absences 


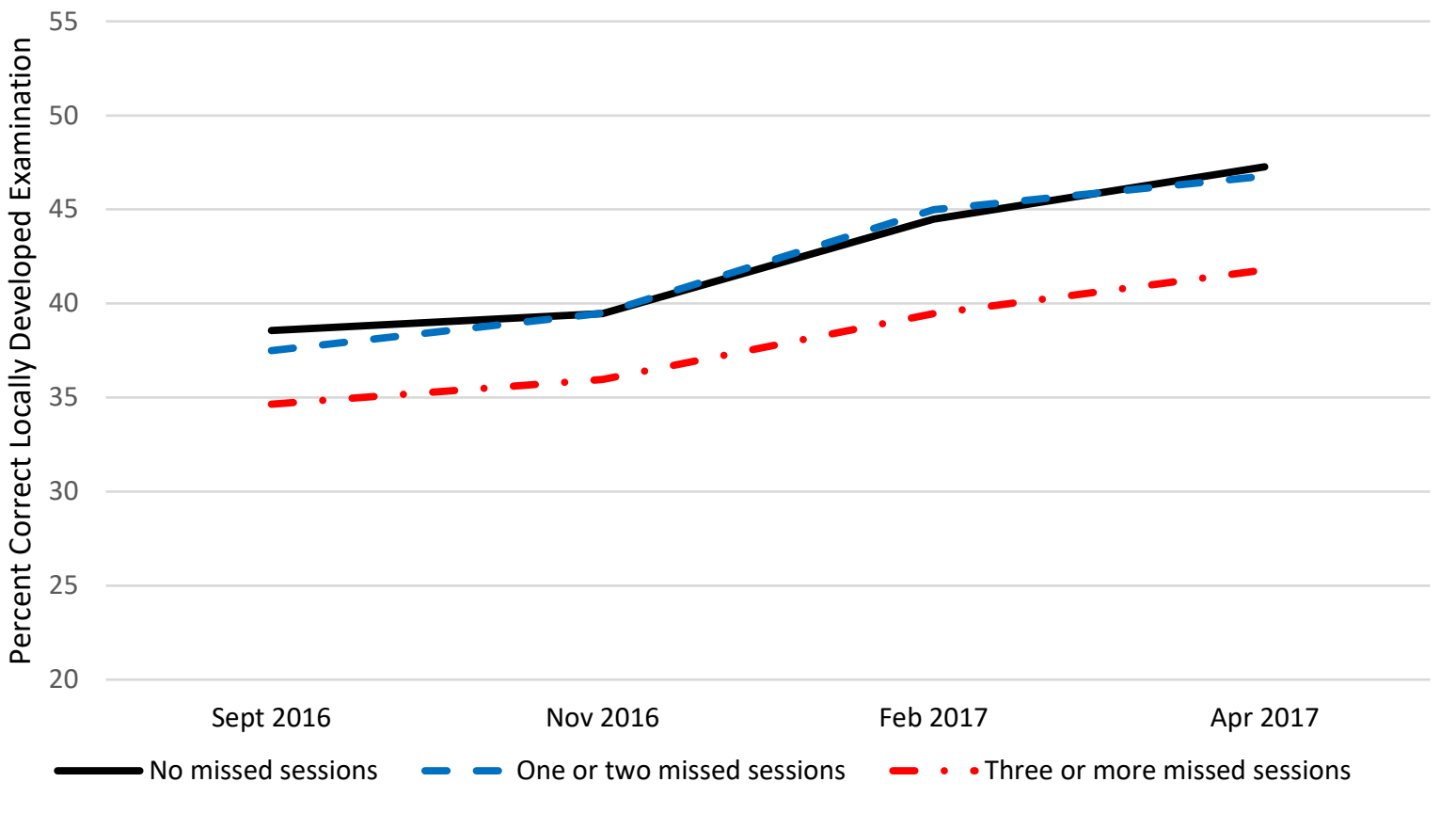




\section{Figure 3 (on next page)}

Clinical skills exam post encounter station performance by number of large group absences 


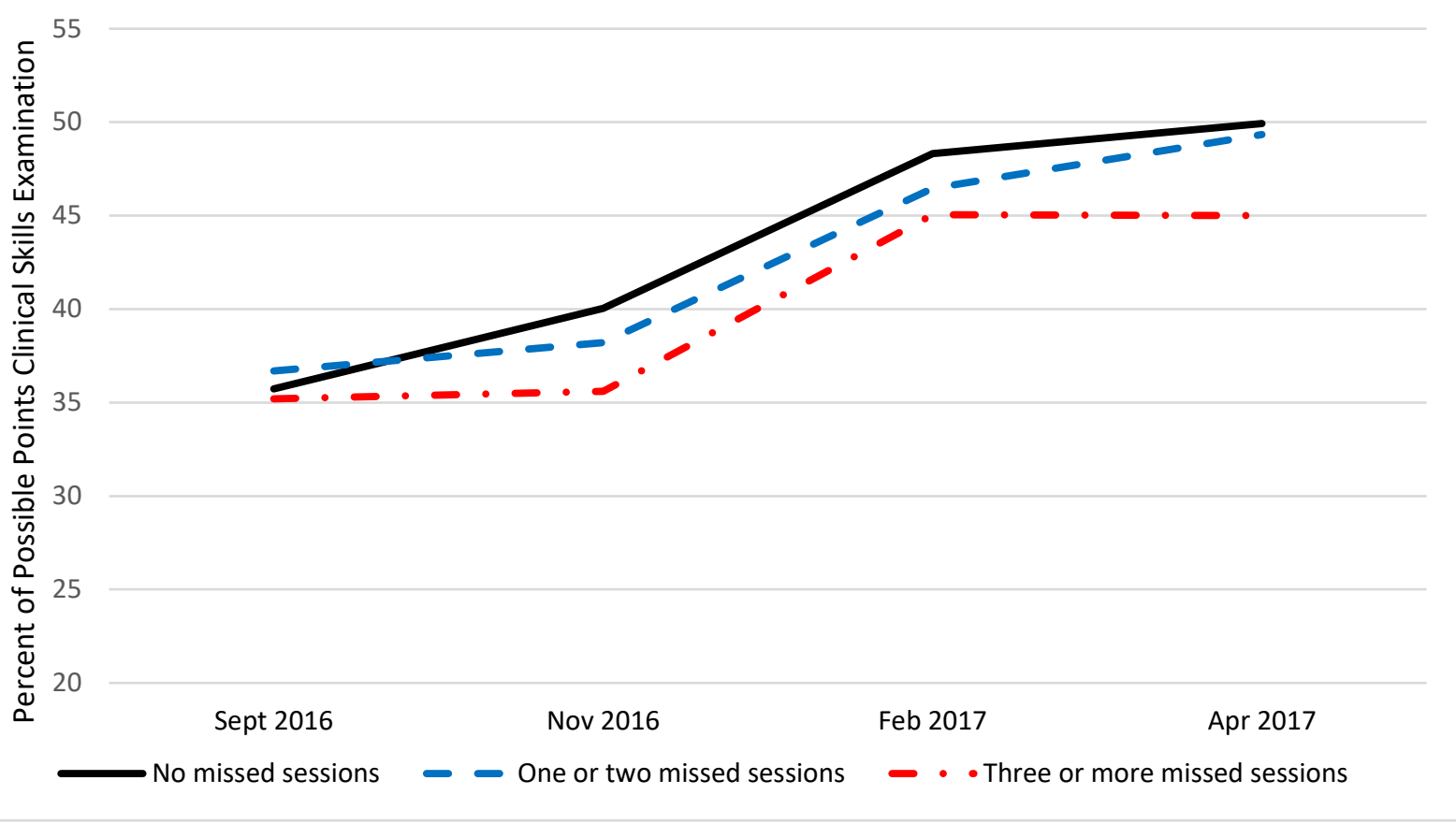




\section{Table 1 (on next page)}

Progress suite of assessment performance by number of missed large group sessions 
2

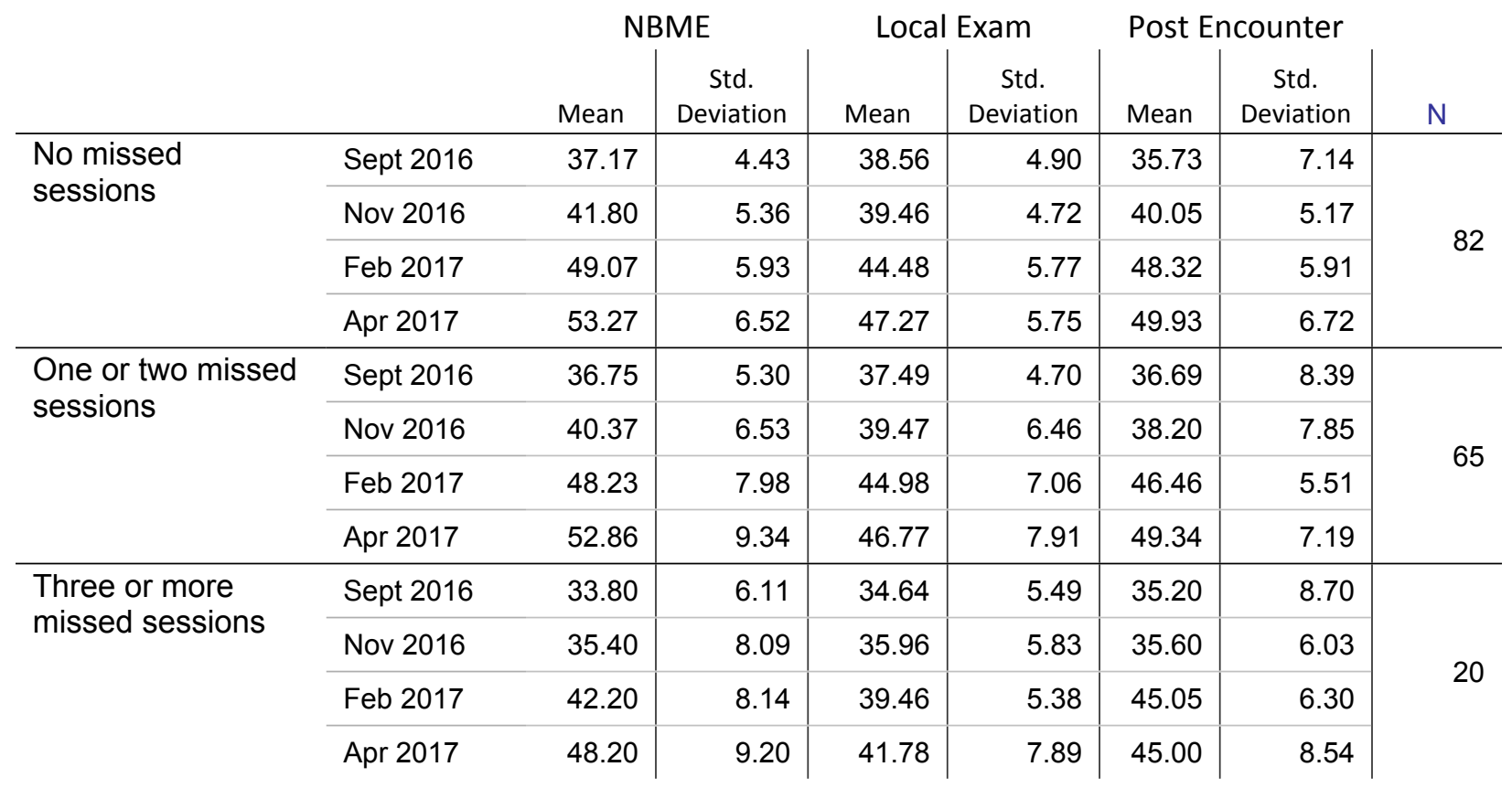

3

4 


\section{Table 2 (on next page)}

Number of assessments at least one SD below the mean by number of missed large group sessions 
1

2

3

4

5

6

7

8

9

10

11

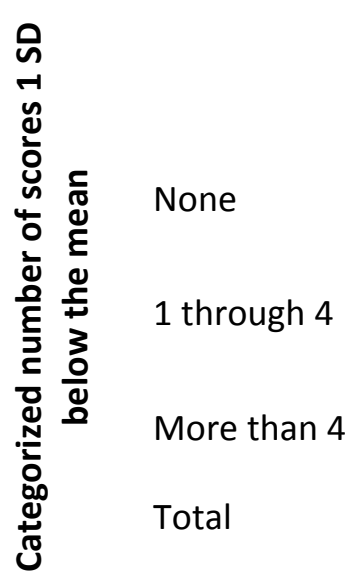

\section{Statistical significance based on Fisher's exact test.*}

No missed sessions vs 1 or 2 missed sessions $p=0.54$

Less than 3 missed sessions vs 3 or more missed sessions $p<0.01$ 